\title{
Development of a psychological intervention for people with bipolar disorder in rural Ethiopia
}

\author{
Mekdes Demissie, Charlotte Hanlon, Lauren Ng, Rosie Mayston, Sisay Abayneh and Abebaw Fekadu
}

\section{Background}

Evidence from high- and middle-income countries indicates that psychological interventions (PSIs) can improve the well-being of people with bipolar disorder. However, there is no evidence from low-income countries. Cultural and contextual adaptation is recommended to ensure that PSIs are feasible and acceptable when transferred to new settings, and to maximise effectiveness.

\section{Aims}

To develop a manualised PSI for people with bipolar disorder in rural Ethiopia.

\section{Method}

We used the Medical Research Council framework for the development and evaluation of complex interventions and integrated a participatory theory-of-change (ToC) approach. We conducted a mental health expert workshop $(n=12)$, four independent ToC workshops and a final workshop with all participants. The four independent ToC workshops comprised people with bipolar disorder and caregivers $(n=19)$, male community leaders $(n=8)$, female community leaders $(n=11)$ and primary care workers $(n=21)$.

\section{Results}

During the workshops, participants collaborated on the development of a ToC roadmap to achieve the shared goal of improved quality of life and reduced family burden for people with bipolar disorder. The developed PSI had five sessions: needs assessment and goal-setting; psychoeducation about bipolar disorder and its causes; treatment; promotion of well-being, including sleep hygiene and problem-solving techniques; and behavioural techniques to reduce anxiety and prevent relapse. Participants suggested that the intervention sessions be linked with patients' monthly scheduled healthcare follow-ups, to reduce economic barriers to access.

\section{Conclusions}

We developed a contextually appropriate PSI for people with bipolar disorder in rural Ethiopia. This intervention will now be piloted for feasibility and acceptability before its wider implementation.

\section{Keywords}

Psychoeducation; behavioural intervention; relapse prevention; individual therapy; theory of change approach.

\section{Copyright and usage}

(C) The Author(s), 2021. Published by Cambridge University Press on behalf of the Royal College of Psychiatrists. This is an Open Access article, distributed under the terms of the Creative Commons Attribution-NonCommercial-NoDerivatives licence (http://creativecommons.org/licenses/by-nc-nd/4.0/), which permits non-commercial re-use, distribution, and reproduction in any medium, provided the original work is unaltered and is properly cited. The written permission of Cambridge University Press must be obtained for commercial re-use or in order to create a derivative work

\section{Burden}

Bipolar disorder is a severe mental illness with an estimated global prevalence ranging from 0.4 to $2.4 \% .{ }^{1}$ In the 2017 Global Burden of Disease Study, bipolar disorder accounted for 9.3 million disabilityadjusted life-years. ${ }^{2}$ In low- and middle-income countries (LMICs), the burden of bipolar disorder is exacerbated by a high treatment gap, ranging from 76 to $90 \%,{ }^{3}$ which contributes to a risk of premature mortality double that of the general population. ${ }^{4}$ In Ethiopia, the lifetime prevalence of bipolar disorder ranges from 0.5 to $1.8 \%{ }^{5,6}$ Studies conducted in different settings in Ethiopia have found that people with bipolar disorder have multiple unmet needs, including a high relapse rate, ${ }^{7}$ premature mortality ${ }^{4}$ and verbal and physical abuse. ${ }^{8}$ In addition, bipolar disorder in Ethiopia is associated with substantial social, physical and functional role restrictions. ${ }^{5}$ Studies conducted in mental and general hospitals in Ethiopia have also reported a higher risk for hospital admission and length of hospital stay among people with bipolar disorder compared with other patients, ${ }^{9,10}$ which is associated with higher healthcare costs ${ }^{9}$ and insufficient use of limited mental health resources. ${ }^{11}$

\section{The unmet psychosocial needs in LMICs}

Studies conducted globally have identified potential mediators and moderators of bipolar disorder treatment outcomes, such as treatment adherence, ${ }^{12}$ knowledge about the disorder ${ }^{13}$ and sleep hygeine. ${ }^{14}$ Moreover, social factors, including stigma and discrimination, ${ }^{15}$ stressful life events ${ }^{16}$ and substance use, ${ }^{17}$ may influence the course and outcome of the disorder. In LMICS, inadequate care for people with bipolar disorder contributes to functional impairment, stigma, discrimination, human rights violence and premature death. ${ }^{18}$ Therefore, to reduce the risk of relapse and improve the well-being of people with bipolar disorder, these factors must be addressed. ${ }^{19}$

The Mental Health Gap Action Programme intervention guide (mhGAP-IG) lists mood stabilisers as the main treatment for bipolar disorder. ${ }^{20}$ However, in many LMICs, these medications are either unavailable or their supplies are unreliable. ${ }^{3}$ As a result, people with bipolar disorder in rural Ethiopia are treated with typical antipsychotic medications, ${ }^{21}$ which are more likely to cause adverse effects. ${ }^{9}$ Studies conducted in Ethiopia have also shown that people with bipolar disorder have unmet psychological, social and treatment-related needs, ${ }^{22}$ and people with mental illness need information about their illness and treatment. ${ }^{21}$

Studies conducted mainly in high-income countries indicate the efficacy of psychological interventions for reducing relapse, hospital admission $^{23}$ and symptom severity. ${ }^{13}$ Evidence-based, contextualised psychological interventions for people with mental illness are scarce in LMICs, including Ethiopia. ${ }^{24}$ Additionally, LMICs typically lack the specialised mental health professionals who could deliver psychological interventions. ${ }^{25}$ In response to this, the World Health Organization recommends task-sharing delivery of mental healthcare with available and affordable non-specialist health professionals, ${ }^{26}$ and integrating the service into primary healthcare $(\mathrm{PHC}){ }^{27}$ In Ethiopia, in line with the National Mental 


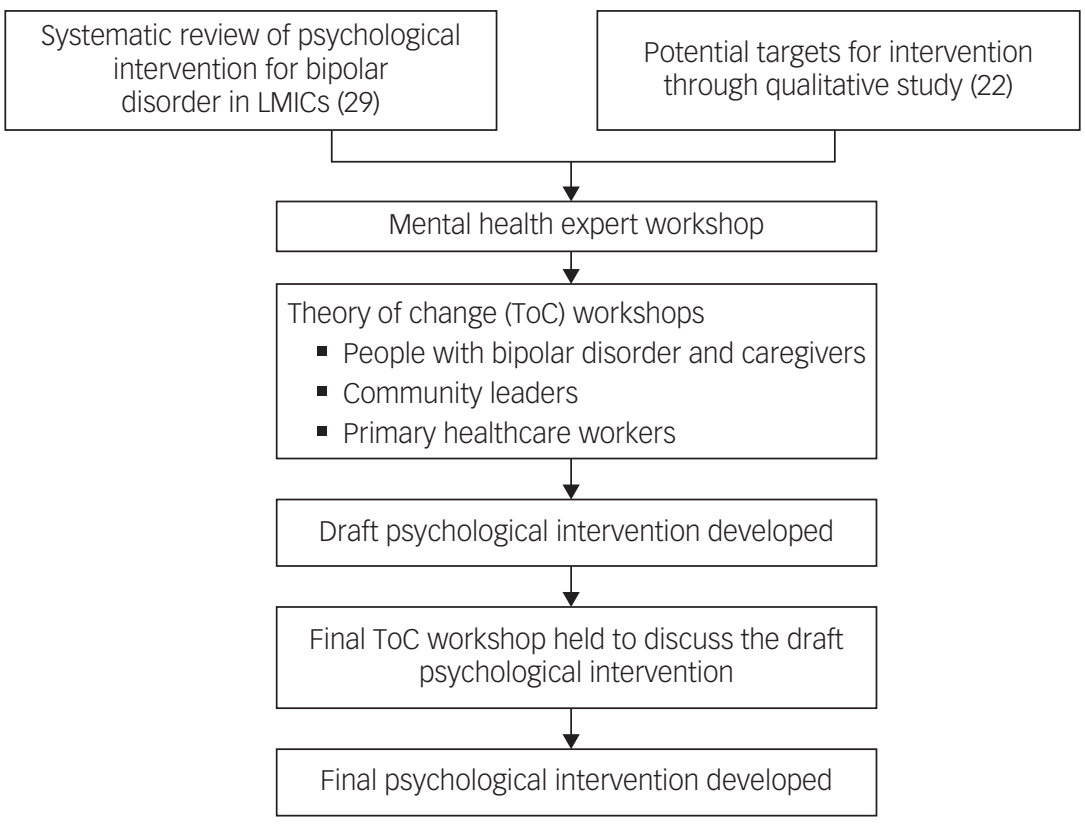

Fig. 1 Overview of the process of development of a psychological intervention for bipolar disorder. LMICs, low- and middle-income countries.

Health Strategy, there have been efforts to scale up primary carebased mental healthcare. As a demonstration project, the Programme for Improving Mental Health Care (PRIME project) has developed and implemented a scalable mental healthcare plan for bipolar disorder based in PHC. ${ }^{28}$ According to a systematic review of non-specialist-delivered mental health interventions in LMICs, psychological intervention have positive effects on depression $^{26}$ - another mood disorder - yet few psychological interventions have been developed for bipolar disorder in LMICs.

A systematic review that included studies from LMICs showed the effectiveness of psychological intervention in improving the outcomes of bipolar disorder. The review included 18 studies, but none of them were from low-income countries and only a single study was found from Africa. The review also reported that only a few of the included studies reported how the interventions were developed or adapted. ${ }^{29}$ The meta-analysis of 76 interventions reported that the interventions that were targeted at a specific cultural group were four times more effective than interventions provided to groups consisting of clients from a variety of cultural backgrounds. ${ }^{30}$ The adaptation of psychological intervention to the target population's culture and social context also plays a critical role in improving the feasibility and acceptability of an intervention. ${ }^{24}$ Psychological interventions that can be delivered by non-specialists may play a particularly important role in settings where there is inadequate access to mood stabilisers and mental health specialists. Therefore, the aim of this study was to develop a contextualised, scalable and manualised psychological intervention for people with bipolar disorder that could be delivered by PHC workers in rural Ethiopia.

\section{Method}

\section{Design}

We used the Medical Research Council (MRC) Framework for the Development and Evaluation of Complex Interventions, integrated with a theory-of-change (ToC) approach. ${ }^{31}$ The MRC framework has four phases - intervention development, feasibility and piloting, evaluation and implementation - all of which take place as an iterative rather than a linear process. We followed four steps to develop the intervention: (a) a systematic review, (b) a qualitative study with people with bipolar disorder and their caregivers, (c) a mental health expert workshop and (d) ToC workshops with various stakeholders (Fig. 1). The findings of the systematic review and qualitative study were published previously, ${ }^{22,29}$ and the key findings are summarised in Table 2 and Supplementary File 1 available at https://doi.org/10.1192/bjo.2021.999.

ToC has been defined as a 'theory of how and why an initiative works, ${ }^{31}$ A ToC roadmap is developed using feedback from stakeholders, and is amenable to change throughout the intervention development and evaluation process. ToC can be incorporated into, and provide practical guidance for, the different phases of the MRC framework. ${ }^{31}$ The ToC approach has been used to develop, implement, monitor and evaluate complex mental health interventions in Ethiopia. ${ }^{28}$

\section{Setting}

The qualitative study and ToC workshops were carried out at two adjacent sites: the Sodo and Butajira districts of the Gurage Zone, Ethiopia. Sodo district is located $100 \mathrm{~km}$ from the capital city of Addis Ababa, and had an estimated total population of 173185 in $2014 .{ }^{32}$ Sodo Gurage is the largest ethnic group (85.3\%), followed by Oromo (11.6\%). Most of its population are followers of Orthodox Christianity (97\%). Butajira district is located $130 \mathrm{~km}$ from Addis Ababa and had an estimated total population of 350297 in 2017, ${ }^{32}$ with most of the population following Islam. The town of Butajira is a Health and Demographic Surveillance Site that has been functioning since 1987.

The Ethiopian healthcare system comprises three levels. The first level, which is the focus of this intervention, consists of PHC, which includes primary hospitals, health centres and health posts. Health centres are staffed with health officers, nurses and midwives. ${ }^{33}$ Health posts are staffed with health extension workers (HEWs), high school graduates with 1 year of training in disease prevention and health promotion activities at the community level. ${ }^{33}$ Sodo district has eight health centres and one primary hospital; Butajira district has one district hospital and 13 health centers. ${ }^{33}$ PHC workers in both districts have been trained and 
supported to deliver mental healthcare through the PRIME project in Sodo district ${ }^{28}$ and the Task-Sharing for the Care of Severe Mental Illness in a low-income country (TaSCS) project, which aims to test the effectiveness and cost-effectiveness of task-sharing the care of people with a severe mental illness within the PHC setting in Butajira. ${ }^{33}$

\section{Participants and data collection methods}

\section{Mental health expert workshop}

Twelve (three female and nine male) health experts and health professionals from diverse professional backgrounds (two psychiatrists, three public health professionals, five clinical psychologists, one pharmacist and one social worker) participated in this workshop. Findings from the systematic review ${ }^{29}$ and the qualitative study ${ }^{22}$ (stages 1 and 2) were presented and discussed. The experts were then asked to give their suggestions and recommendations on the content and delivery of the intervention based on the systematic review, the qualitative findings and their clinical and research experience. The workshop was conducted in Amharic and English in Addis Ababa, and lasted for 3 h. A.F., a psychiatrist, facilitated the discussion. The discussion was audio-recorded and notes were taken by the first author.

\section{ToC approach}

To reduce power imbalances among ToC workshop participants, we initially conducted separate workshops with groups of stakeholders with the lower difference among them. ${ }^{34}$ Additionally, we engaged a balanced number of participants from each stakeholder groups to ensure the representation of various stakeholders' voices: (a) people with bipolar disorder and their caregivers, (b) women community leaders, (c) male community and religious leaders, and (d) PHC workers and district-level government office personnel. Even within these broad categories, power imbalances exist, e.g. between people with bipolar disorder and caregivers or between PHC workers and district-level government office personnel. In this setting, people with bipolar disorder commonly rely on caregivers to support them to attend meetings, and so the presence of both caregivers and people with bipolar disorder was necessary. To address this concern, within workshops, the facilitators paid particular attention to ensuring that all voices were heard. We grouped PHC workers and government officials in one ToC workshop because most of the officials were health professionals and had experience working in a PHC setting. Additionally, PHC workers and district-level officials have a culture of working together for a common purpose. The final ToC workshop included all participants from the stakeholder groups to ensure that each group could hear the others' voices, and to discuss issues that needed consensus.

The ToC workshop participants were selected purposively based on their experience in mental healthcare service delivery or roles in traditional or social associations in the communities. The first four ToC workshops were conducted in the Sodo district in August 2019, and the last ToC workshop was held in Butajira town in September 2019.

All ToC workshops were co-facilitated by M.D. and S.A., both of whom are $\mathrm{PhD}$ students. S.A. has previous experience in facilitating ToC workshops with various groups in the study site. All participants were given an opportunity to express their opinions, and were encouraged to identify challenges and suggest possible solutions for each topic area. The facilitators summarised the discussions, recorded key points on a flip chart and asked for confirmation. All ToC workshops except the final one, had two sections: (a) exploring the feasibility and acceptability of a psychological intervention; and (b) developing a ToC map that indicated the causal pathway through which the proposed psychological intervention was expected to achieve consensus in its short-, medium- and long-term effects, identification of preconditions for achieving the effect, possible barriers and facilitators and indicators of success.
In the first section, findings from the systematic review and qualitative study were presented, participants were introduced to the objectives of the ToC workshop, and their expected roles were outlined in a Microsoft PowerPoint for Windows presentation prepared in Amharic. Participants then discussed the findings, the potential benefits of psychological intervention, and the feasibility and acceptability of this approach. The participants were asked to consider anticipated or experienced responses to the intervention in relation to the culture and religion of the society; health system resources; the impact of the socioeconomic status of people with bipolar disorder and their caregivers; and the community, transportation availability, affordability, accessibility of the healthcare service, the PHC workers' time and any other relevant considerations.

During the second section, we used farming metaphors to explain the ToC concepts, procedures and avoid technical terms. For example, we asked question like: What did the farmer wants to achieve (effect)? What do farmers do to prepare the land for farming (interventions/pre-conditions)? Which activities should be done first and last, and why? The participant responses were used to explain how a similar way of thinking can help us to plan the development and testing of psychological intervention.

The actual ToC workshop then started by asking the participants what they wanted to see in the long run for people with bipolar disorder. Short-, medium- and long-term outcomes and wider effects were agreed upon, and then the pathways, interventions, preconditions, assumptions and indicators of success were mapped using sticky notes posted on the wall. An integrated ToC map was drafted by reviewing the ToC maps arising from each of the four workshops alongside the minutes and the recorded discussions. The goal of the fifth and final ToC workshop was to bring all stakeholder groups to a consensus on the content, timing, duration and number of sessions of the intervention, including implementation strategies to overcome potential barriers, in the context of broader care for people with bipolar disorder. In an initial presentation, the shared and divergent ideas from the preceding workshops were summarised and used to prioritise discussion points.

All ToC workshops were conducted in Amharic, lasted an average of $5 \mathrm{~h}$, were audio-recorded and minutes were taken. The minutes, drafted ToC maps and recorded ToC workshops were reviewed by M.D. and A.F. to refine the ToC map and finalise the content of the psychological intervention. The draft manual was translated into the local language, and PHC workers reviewed and approved the content for pilot testing.

\section{Analysis}

All audio recordings were transcribed verbatim in Amharic and then translated into English. The transcripts were imported to OpenCode version 4.03 for Windows (Umeå University, Umeå, Sweden; see https://www.umu.se/en/department-of-epidemiologyand-global-health/research/open-code2/) to facilitate data management and assist analysis. We used thematic analysis procedures. First, MD conducted line-by-line coding of all five ToC workshop transcripts and shared them with the second author (C.H.) for review. M.D. and C.H. mapped codes onto themes deductively based on key components of the ToC map, including key tasks, intervention, preconditions, assumptions and indicators, as well as acceptability and feasibility (Table 3). Finally, we summarised the findings in the tables and text, and identified illustrative quotes.

In each $\mathrm{ToC}$ workshop, four draft ToC maps were developed through discussion and consensus, and then combined by the authors to develop a single ToC map. Finally, this final draft ToC map was refined and approved in the fifth ToC workshop with all ToC workshop participant groups. 
We triangulated the findings with different information sources (Table 2) to increase trustworthiness. Furthermore, M.D. and S.A. were engaged for an extended time in the field. The involvement of co-authors with multidisciplinary backgrounds improved data interpretation.

\section{Quality of reporting}

We used the Guidance for Reporting Intervention Development (GUIDED) checklist $^{35}$ to report the intervention development process.

\section{Ethical approval}

The Institutional Review Board of the College of Health Sciences of Addis Ababa University approved the study (reference number 043/ $17 /$ Psy), and written informed consent was obtained from each participant.

\section{Results}

\section{Mental health expert workshop}

Mental health experts suggested two intervention components: (a) components that could be used to improve the PHC workers' skills in establishing therapeutic rapport and competence in needs assessment and goal-setting; and (b) the 'active ingredients' of the intervention, such as psychoeducation, development of relapse prevention plans and behavioural techniques such as muscle relaxation and breathing exercises, to help people with bipolar disorder to control their overarousal, aggression and anxiety symptoms. Experts recommended four intervention sessions for the following reasons: in our systematic review, most of the included studies reported three to eight sessions; ${ }^{29}$ previously, four to eight sessions of interpersonal therapy for common mental disorders were proposed for a PHC setting in Ethiopia; ${ }^{36}$ and the proposed components of intervention could be covered in four sessions. Participants suggested that PHC workers should deliver the intervention based on their being trained on the mhGAP-IG and having experience of treating people with severe mental illness. However, they were concerned about the potential work burden on PHC workers.

Experts recommended an individual format to simplify the challenge of having groups at a set time because of transportation problems in rural settings and other factors. They also recommended that caregivers be involved in the intervention considering the sociocultural context in which family members accompany patients when attending the facility. They reasoned that caregivers' involvement in the intervention could help to create awareness about the illness in the wider family as well as support their ability to help their family members. Nonetheless, they emphasized that people with bipolar disorder should decide whether their family members should be involved. Finally, to facilitate intervention provision and create awareness for people with bipolar disorder and their caregivers, they suggested the development of an illustrated information leaflet that explains the causes, symptoms and treatment of bipolar disorder.

\section{Results of the ToC workshops}

Four independent ToC workshops and one ToC workshop that included all participants were conducted, with a total of 59 participants (Table 1).

\section{Feasibility and acceptability of psychological intervention}

Participants perceived that PSI was needed for people with bipolar disorder. However, they also argued that the developed intervention must be feasible and acceptable to be implemented. The group that

\begin{tabular}{lccc} 
Table 1 Theory-of-change workshop participants & & \\
Stakeholder group & Female & Male & Total \\
ToC with patients and caregivers & & & \\
$\quad$ People with bipolar disorder & 4 & 4 & 8 \\
$\quad$ Caregivers & 6 & 5 & 11 \\
ToC with male community leaders & - & 8 & 8 \\
ToC with female community leaders & 11 & - & 11 \\
ToC with professionals & & & \\
$\quad$ Primary healthcare workers & 5 & 11 & 16 \\
$\quad$ District-level government office representative & 0 & 5 & 5 \\
Final ToC workshop participants & 26 & 33 & 59 \\
Toc, theory of change. & & & \\
\hline
\end{tabular}

included people with bipolar disorder and their caregivers described the varying nature and intensity of symptoms over time. Because of the episodic nature of the illness, bipolar disorder was perceived by many people with bipolar disorder and their caregivers to be caused by evil spirits or other supernatural acts, leading them to try various religious or traditional treatments. They also noted the chronicity of the illness and the need for long-term support:

'... Mental illness is not like tuberculosis, which gets cured just by giving medication. Mental illness requires long-term support and effort from doctors, caregivers and the surrounding societies ...'

People with bipolar disorder and caregivers reported that people with bipolar disorder are sensitive; simple stressors can be enough to trigger their illness. When other people speak of their own social affairs, they may be suspicious and assume that they are the focus of discussion, which may hurt them psychologically. Community leaders also noted that medications are important to people with bipolar disorder, and they believe that caregivers had a responsibility to support access. People with bipolar disorder and their caregivers reported experiencing stigma because of having a mental illness or having a relative with mental illness. One caregiver said:

'... At the coffee shop, at a wedding, there are people who treat [the person with bipolar disorder] as if she is a different person. In such situations, there are times that she would return to home because her feelings get hurt ...'

The intervention was perceived as important because it would provide information about bipolar disorder, its causes and treatments, and would decrease misconceptions. One participant said:

'... People take patients to different traditional places because of lack of awareness. As long as society is well-informed about the intervention and where they can access it, they will go to the health facility as soon as they feel sick. For instance, if someone gets malaria the society is well-informed about where to get treatment and the same is true in this case'.

The health professionals also expected that the intervention would be acceptable for PHC workers because it would help them develop their skills and would improve the acceptability of the service. This, in turn, was predicted to improve the mental health knowledge of people with bipolar disorder, their families and the community, and increase treatment adherence and improve outcomes. Health professionals reflected that, often, people with bipolar disorder visit health facilities only after the illness become severe. They emphasised that early detection of relapse should be addressed in the intervention.

Participants in all groups considered the health centre as the ideal place for the intervention because this is where people with bipolar disorder receive their regular follow-ups and fill their prescriptions. Additionally, a quiet and private place was preferred, but without segregating people with bipolar disorder from other 


\section{Methods}

Systematic review

Qualitative study

Mental health expert workshop

Theory-of-change workshops

\section{Findings}

- Identified psychological intervention: psychoeducation, family therapy, cognitive-behavioural therapy and mindfulness-based cognitive therapy

- The number of sessions ranged from 1 to 18

- The content of the intervention includes education about signs and symptoms of bipolar disorder, the causes and prognosis of bipolar disorder, treatment adherence and sideeffects of medication, early identification of symptoms of relapse, triggering factors, substance use, regular habits and management plans or prevention strategies

- Intervention providers were mental health specialists or practitioners

- People with bipolar disorder and their caregivers reported perceiving early signs and symptoms of relapse

- A major concern for people with bipolar disorder and their caregivers related to the patients' illness being identified

- Perceived factors that precipitate or worsen the illness were explored

- Coping mechanisms used by people with bipolar disorder to cope with stressful life events were explored

- Bipolar disorder has a negative effect on the social, functional and economic status of people with bipolar disorder and their families

- Possible components of the intervention were suggested to address the concerns of people with bipolar disorder and improve their health and treatment outcome

- Stigma and financial problems

- Need for psychological intervention

- The necessary condition for improving the acceptability and feasibility of psychological intervention

- Developed theory-of-change map

- Need for training in communication skills for intervention providers

- Need to improve community awareness
Contribution to the psychological intervention development

Defined the type of intervention

- Defined the intervention content

- The studies were used to identify the intervention manual

- Define problems from the people with bipolar disorder and caregivers' lived experience

- Identify psychosocial factors that could be addressed in the current psychological intervention

- Experts suggested intervention components based on their clinical and research experience as well as findings of qualitative and systematic review

- Explored the feasibility and acceptability of psychological intervention

- Defined the necessary resources to give the intervention

- Support the patients to use the existing supporting platforms like the safety net programme

- Suggested the intervention content, frequency of sessions, format, and providers

- Defined the desired outcome

- Defined indicators for success health centre attendees, to avoid stigma and discrimination. Some participants from the community and health professionals suggested that the intervention should be located at the health post to increase accessibility, and it should be delivered by the health centre staff as part of outreach activity. Finally, participants in the final ToC workshop agreed that the intervention should be delivered at the health centre by PHC workers because PHC clinicians are in a better position to know the mental health history and current health status of the person with bipolar disorder, and this will improve trust because the clinician is likely to be known to them from their routine care. Participants also mentioned the importance of HEWs creating awareness in the community, in the form of a campaign or through another mechanism, as an important supportive activity.

The participants came to a consensus that a one-to-one consultation format would be better than a group format. Among the reasons, people with bipolar disorder may not want to talk about their social, economic and personal lives in front of others. Furthermore, in rural areas, people may struggle to attend group interventions at a specific time. Similar to the mental health experts, participants also suggested the option of a common session for people with bipolar disorder and their caregivers as long as the patients are willing and it does not interfere with their privacy:

'People do not come alone and will have someone with them, those that came will also get an education on the subject that they will later transfer to other family members and improve the support they provide to the patient'.
Regarding the session, people with bipolar disorder and their caregivers and community groups suggested that the number, duration and frequency of intervention sessions should be determined based on the content and advice of professionals. Additionally, they underlined the importance of aligning the monthly intervention sessions with regular appointment dates, to encourage attendance by minimising transportation costs and time. PHC workers provided different suggestions for the duration of session (from 20 to 45 min). Finally, considering the workload of PHC workers, they reached a consensus to reduce the intervention content per session to be covered in a maximum of $20 \mathrm{~min}$ and to increase the number of sessions from four to five. They also suggested working in collaboration with HEWs, especially to help people with bipolar disorder with their treatment adherence. The PHC workers raised the issue of workload and expressed concerns about people with bipolar disorder and their caregivers being made to wait for a long time while they delivered the intervention.

All participants agreed on the importance of preparing an information leaflet to facilitate the sessions and encouraging the participants to share the information with their entire families and neighbours. The key findings and contributions of various methods to the development of the intervention is summarised in Table 2 .

\section{ToC Map}

Factors identified as necessary to the development and implementation of a psychological intervention for people with bipolar disorder are summarised in the ToC map (Fig. 2) and described below. 


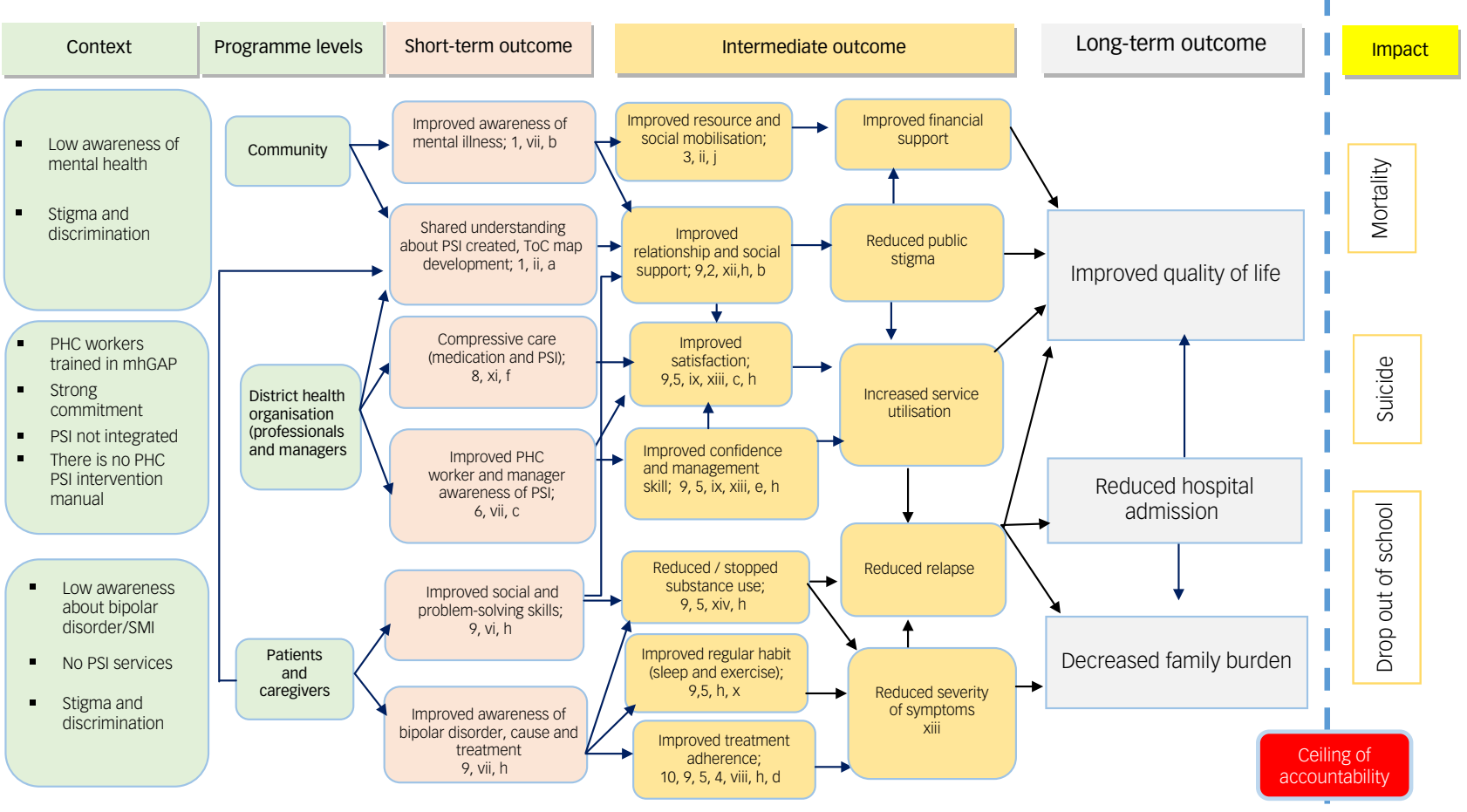

Fig. 2 ToC map for the development of a psychological intervention for bipolar disorder in rural Ethiopia. Example of assumptions: willingness and motivation to (1) be involved in ToC workshop; (2) work with PHC workers; (3) mobilise resources; (4) deliver PSI as per the manual; (5) give compassionate and respectful care; (6) undergo theoretical and practical training on PSI; (7) supervise, monitor and support PHC workers; (8) make the PSI manual available at the health facility; (9) people with bipolar disorder/caregivers receive all components of the PSI and (10) offer community and family support. Examples of indicators: number of stakeholders involved in (i) awareness creation programme, (ii) ToC workshop, (iii) resource mobilisation, (iv) type and amount of resource mobilised, (v) number of PHC workers who attended PSI training, (vi) number of caregivers/people with bipolar disorder who attended one session and four sessions, (vii) $80 \%$ increase in awareness, (viii) decreased severity of signs and symptoms as measured with the YMRS and PHQ, (ix) number of participants satisfied with treatment as assessed by an indepth interview, $(x)$ number of patients with a regular habit, (xi) number of health facilities that made the PSI manual, (xii) social inclusion, (xiii) number of professionals satisfied and (xiv) reduced substance use as measured by ASSIST. Example interventions: (a) conduct ToC workshop, (b) create mental health awareness creation programme, (c) offer theoretical and practical PSI training for PHC workers and managers, (d) engage people with bipolar disorder and caregivers in treatment planning, (e) ensure medication availability at the health centres, (f) make the PSI manual available at the health centres, $(g)$ support patients in adhering to treatment, (h) deliver psychological intervention for people with bipolar disorder and caregivers, (i) evaluate the intervention and (j) mobilise resources. ASSIST, The Alcohol, Smoking, and Substance Involvement Screening Test; mhGAP, Mental Health Gap Action Programme; PHC, primary healthcare; PSI, psychological intervention; SMI, severe mental illness; ToC, theory of change; YMRS, Young Mania Rating Scale.

\section{Outcomes and effects}

People with bipolar disorder and their caregivers mentioned improved social and psychological well-being and reduced family burden, hospital admission and school drop-out as the desired long-term outcomes of the intervention, with reduced mortality as a potential broader effect of the intervention. Community leaders focused on stigma reduction and improved physical, social and functional well-being as the preferred long-term outcome. PHC workers and district health office managers emphasised the improved quality of life of people with bipolar disorder and reduced family burden as a long-term outcome. Participants also mentioned reduced mortality and disability related to the illness as a desired effect, but recognised that these require multisectoral changes and are not expected to be achieved just through the psychological intervention alone. In the final ToC workshop, participants discussed the feasibility of the identified long-term outcomes and reached a consensus that reduced hospital admissions, reduced caregiver burden and improved quality of life would serve as the long-term outcomes. Participants also discussed and agreed that the reduction of mortality and school drop-out needs the involvement of various stakeholders beyond the delivery of psychological intervention. As a result, they reached a consensus that reduced mortality and school drop-out would serve as an effect.

\section{Preconditions for intervention}

Participants were asked to list the interventions needed, preconditions, assumptions and indicators. Participants mentioned that there should be interventions for people with bipolar disorder and their caregivers, implemented at the community and health facility level. Likewise, the preconditions, assumptions and indicators were also identified to achieve an agreed-upon outcome were also identified, as illustrated in Table 3

\section{Description of the newly developed intervention manual}

The findings from the formative qualitative study and ToC workshops were triangulated to identify the unmet needs and priorities of people with bipolar disorder. These inputs were then used to select the intervention components and to decide on the number, frequency and duration of sessions, as well as the facility where the intervention should be provided. During the design of the intervention, the identified needs and priorities were linked to the components of the intervention (Table 4).

In general, the intervention manual was structured in five sessions: (a) needs assessment and goal-setting; (b) psychoeducation about bipolar disorder, its causes and influencing factors; (c) treatment and treatment adherence; (d) sleep hygiene and 


\section{Table 3 Summary of level of key tasks, intervention, preconditions, assumptions and indicators}

\begin{tabular}{|c|c|c|c|}
\hline $\begin{array}{l}\text { Levels of } \\
\text { intervention }\end{array}$ & Key tasks & Intervention & Preconditions \\
\hline $\begin{array}{l}\text { Patients with } \\
\text { bipolar } \\
\text { disorder }\end{array}$ & $\begin{array}{l}\text { - } \quad \text { Identify early signs and } \\
\text { - } \quad \text { Actively engage in their } \\
\text { own treatment plan } \\
\text { - } \quad \text { Practise having a regular a } \\
\text { habit (e.g. sleep) } \\
\text { - } \quad \text { Use behavioural } \\
\text { techniques such as } \\
\text { breathing exercises and } \\
\text { muscle relaxation } \\
\text { - } \quad \text { Practise problem-solving } \\
\text { skills in their daily lives } \\
\text { - } \quad \text { Prepare a relapse } \\
\text { prevention plan }\end{array}$ & $\begin{array}{l}\text { - } \text { Psychoeducation } \\
\text { - } \text { Teaching behavioural } \\
\text { - } \text { techniques } \\
\text { techning problem-solving } \\
\text { - } \text { Awareness of regular } \\
\text { habits } \\
\text { - Involvement in the } \\
\text { development of PSI ToC } \\
\text { map }\end{array}$ & $\begin{array}{l}\text { People with bipolar disorder } \\
\text { - Improved awareness } \\
\text { about the illness, cause } \\
\text { and treatment } \\
\text { - } \quad \text { Learned and practised } \\
\text { behavioural techniques } \\
\text { - Improved adherence to } \\
\text { - PSI and for medication } \\
\text { - } \quad \text { Practised a regular habit } \\
\text { - Improved health-seeking } \\
\text { - } \text { impehaviour } \\
\text { - } \text { relapse preved awareness of a }\end{array}$ \\
\hline
\end{tabular}

- Psychoeducation

- Teaching behavioural techniques

- Training on problemsolving techniques and regular habits

- Involvement in PSI TOC map development

- Active participation in treatment and relapse prevention plan, and help people with bipolar disorder
Caregivers

- Improved their awareness about the illness, its cause and treatment

- Improved involvement in the treatment plan

- Improved practise in supporting people with bipolar disorder

\section{- Working with the community and religious leaders, and HEWs \\ - Awareness about mental illness in general and bipolar disorder in particular \\ Community - Mobilisation of resources \\ - Support patients and caregivers \\ - Care for and love for patients and caregivers \\ - Helping patients with social integration}

\section{Assumptions}

- The intervention is feasible and acceptable

- People with bipolar disorder

- $\quad$ Are able to attend all sessions

- Discuss and practise what they have learned in the training

- Motivated to take an assignment
Indicators

- Number of patients and caregivers attending the first and all sessions

- Duration of each session

- Number of patients adherent to treatment

- None/mild mood symptoms based on the YMRS and PHQ

- $80 \%$ increase in awareness of bipolar disorder and its causes, treatment and prevention plan

- Number of participants satisfied with the intervention

- Number of patients with a regular habit compared with baseline

- Level of social support received as measured by OSLO

- Number of patients and caregivers attending first and all sessions

feasible and acceptable

Caregivers

- Improved community awareness about bipolar disorder, its causes and treatments

- Improved communication among patients, caregivers and intervention providers

- Community nonstigmatising attitudes and support

- Improved collaboration of community stakeholders

Health facility Made the psychological (service intervention service provider/ available with the required manager) quality

- Compassionate and respectful care for patients with bipolar disorder

- Involve patients and caregivers in treatment planning

- PHC workers and patients/ caregivers prioritise problems for the patients/ caregivers need to solve.
- Giving both theoretical and - Improved knowledge of practical training on PSI for PHC workers

- Engaging patients and caregivers in treatment planning

- Ensuring medication availability for bipolar disorder at the health centres

- Adhering to intervention manual when treating patients with bipolar disorder and skills in PSI

- Improved confidence in giving PSI

- Intervention provided as per the manual

- Improved medication availability encouraged patients and caregivers to get involved in the treatment planning
- PHC workers having
- Able to attend all sessions

- Willing and motivated to help people with bipolar disorder

- Willing to work with PHC workers

- Non-stigmatised and nonstigmatising to patients

- Duration of each session

- $80 \%$ increase in caregiver awareness of bipolar disorder, its cause and treatment

- Number of caregivers satisfied with intervention

- Level of social support received as measured by OSLO

- Number of community members with awareness of bipolar disorder, its causes and treatment

community leaders are willing to work togethe oward stigma reduction

The community engages in resource mobilisation - Number of community to support people with mental illness not stigmatise those services

- There is a newly developed, feasible and acceptable PSI available for use

Professionals are trained on the PSI manual

- PHC workers are trained in mhGAP intervention guide

- Health facility managers support PHC workers when needed

- PHC workers are motivated to give the PSI members participati support people with mental illness

- Number of PHC workers in the facility who are trained to give PSI

- Quality of therapeutic relationship between patients and $\mathrm{PHC}$ workers, measured by $\mathrm{HAQ}$ manual, measured by ENACT

- Use of the newly developed PSI manual by PHC workers
- Level of adherence to
- The community does who use mental health resource mobilisation to problem-solving techniques to promote well-being and; and (e) behavioural techniques to target anxiety and relapse prevention, and closing session(Table 4). Each session was intended to last for $20 \mathrm{~min}$ and be delivered every month, aligned with the person's attendance for routine care. The intervention was designed to be given by PHC workers who had been trained in the mhGAP-IG and had received 1 week of theoretical and 1 week of practical training. 
Table 4 Intervention components and expected outcomes

\begin{tabular}{|c|c|c|c|c|}
\hline Session & $\begin{array}{l}\text { Unmet needs/priorities of PBD identified } \\
\text { in qualitative study theory-of-change } \\
\text { and mental health expert workshops }\end{array}$ & Sessions & Contents included in session & Reference manual \\
\hline Session one & $\begin{array}{l}\text { - Therapeutic techniques } \\
\text { - Need assessment and goal-setting }\end{array}$ & $\begin{array}{l}\text { Needs assessment and goal- } \\
\text { setting }\end{array}$ & $\begin{array}{l}\text { Therapeutic skills in psychological } \\
\text { intervention } \\
\text { Introduction and checklist } \\
\text { - } \quad \text { Need assessment } \\
\text { - Goal-setting }\end{array}$ & $\begin{array}{l}\text { Where There Is No } \\
\text { Psychiatrist: A Mental } \\
\text { Health Care Manual }\end{array}$ \\
\hline Session two & $\begin{array}{l}\text { - Low awareness of bipolar disorder and } \\
\text { its causes } \\
\text { - PBD are concerned about the long- } \\
\text { term nature of the illness } \\
\text { - Substance use }\end{array}$ & $\begin{array}{l}\text { - What do I need to know } \\
\text { about my illness? }\end{array}$ & $\begin{array}{l}\text { Psychoeducation } \\
\text { - } \quad \text { Sign and Symptoms of bipolar } \\
\text { disorder } \\
\text { - Identification of early } \\
\text { - } \quad \text { Cymptoms of relapse } \\
\text { - }\end{array}$ & $\begin{array}{l}\text { Clinicians' Treatment } \\
\text { Manual for Family- } \\
\text { Focused Therapy for } \\
\text { Early-Onset Youth and } \\
\text { Young Adults }\end{array}$ \\
\hline Session three & $\begin{array}{l}\text { - } \text { Misperception about treatment (e.g. } \\
\text { expecting cure with medication) } \\
\text { - } \quad \text { PBD are concerned about the long } \\
\text { duration of treatment } \\
\text { - Treatment non-adherence } \\
\text { - } \quad \text { Caregivers are concerned about } \\
\text { aggressive behaviours during relapse }\end{array}$ & $\begin{array}{l}\text { - How can the treatment } \\
\text { help me to get well? }\end{array}$ & $\begin{array}{l}\text { Psychoeducation } \\
\text { - Treatment } \\
\text { - Treatment adherence } \\
\text { - Problem-solving techniques } \\
\text { - PBD who are non-adherent }\end{array}$ & $\begin{array}{l}\text { Psychoeducation Manual } \\
\text { for Bipolar Disorder }{ }^{39}\end{array}$ \\
\hline Session four & $\begin{array}{l}\text { - Caregivers are concerned about sleep } \\
\text { problems } \\
\text { - PBD use various negative coping } \\
\text { mechanisms such as using substances } \\
\text { to feel well and improve their } \\
\text { socialisation } \\
\text { - PBD and caregivers identified social, } \\
\text { treatment-related and substance use } \\
\text { issues as triggering factors for the } \\
\text { person's illness } \\
\text { - Social and relationship problems } \\
\text { - Self- and public stigma } \\
\text { - Disagreement and lack of social } \\
\text { support }\end{array}$ & $\begin{array}{l}\text { - What kind of techniques } \\
\text { and activities help me to } \\
\text { improve my health? }\end{array}$ & $\begin{array}{l}\text { Promoting well-being } \\
\text { - Sleep hygiene and daily } \\
\text { routine } \\
\text { - Problem-solving techniques }\end{array}$ & $\begin{array}{l}\text { Cognitive Therapy for } \\
\text { Bipolar Disorder: A } \\
\text { Therapist's Guide to } \\
\text { Concepts, Methods } \\
\text { and Practice } e^{40}\end{array}$ \\
\hline Session five & $\begin{array}{l}\text { - PBD identified anxiety symptoms as } \\
\text { early sign of relapse } \\
\text { - Heavy-headedness } \\
\text { - Anxiety } \\
\text { - Irritability } \\
\text { - Some PBD and caregivers identified } \\
\text { early symptoms of relapse }\end{array}$ & $\begin{array}{l}\text { - What helps me to feel well } \\
\text { when I feel anxious or } \\
\text { stressed? } \\
\text { - What can I do when I } \\
\text { identify early symptoms of } \\
\text { illness? }\end{array}$ & $\begin{array}{l}\text { - Behavioural techniques } \\
\text { - Muscle relaxation } \\
\text { - Breathing exercise } \\
\text { - Relapse prevention plan }\end{array}$ & \\
\hline
\end{tabular}

The manuals and leaflets were translated into Amharic by two clinical psychologists with experience of working with people with mental illness. Mental health experts and PHC workers involved in the ToC workshop reviewed the translated manual and gave feedback that helped to simplify the manual's structure and readability. They recommended that illustrations in the manual and leaflet to be prepared based on local realities; for example, to include false banana trees, which are very common in the study area, as well as pictures representing people from different religions and genders. Case stories were also prepared and annexed to enable PHC workers to engage better and use them as illustrations as needed.

\section{Discussion}

This is the first manualised psychological intervention guide to be deployed in an integrated care context for people with bipolar disorder. We have used a comprehensive approach, including systematic reviews, ${ }^{29}$ local constructs and the ToC approach integrated with the MRC framework. ${ }^{31}$ The participatory development process of the intervention, anchored in ToC, enabled us to clarify how the intervention and associated implementation strategies would contribute to valued long-term goals, as well as the other elements of care that are necessary preconditions to success.
We used qualitative exploration to understand the unmet needs and priorities of people with bipolar disorder and their caregivers that could potentially be addressed by psychological interventions. Priorities identified by primary beneficiaries are important because psychological interventions work through common factors, such as the therapeutic alliance, positive expectations and a convincing treatment rationale, as well as through treatment-specific components. ${ }^{41}$ Therefore, the components of psychological intervention and the cultural adaptation of the intervention to the local context are vital for improving an intervention's effectiveness.

We used ToC approaches involving different stakeholders, including people with bipolar disorder and their caregivers, community leaders and health professionals. This participatory approach was useful to understand the context and make decisions that reflect scientific evidence the views of patients/caregivers and service providers, to develop and implement the intervention. Therefore, the ToC approach worked as a bridge between the evidence and the local context, helping to ensure ownership, acceptability and support for the intervention, which are key to its implementation. The participatory approach also helped to build trust, encouraging the pooling of resources and knowledge. ${ }^{42}$

In our mental health expert and $\mathrm{ToC}$ workshops, psychoeducation about bipolar disorder, its causes, treatment and the course of the illness were considered essential by all groups of participants. 
This was seen as the key to improving the knowledge and attitudes of people with bipolar disorder, and their caregivers helping them to understand the illness and its treatment. ${ }^{29}$ In this rural setting, literacy levels and access to formal education (beyond primary) are low, and most people lack access to the internet, underscoring the value of including consistent, relevant and evidence-based information within the intervention.

In the ToC workshop, participants spoke of the importance of caregivers' support and endorsed the importance of involving them in the intervention. Previous studies also mentioned that family members are the primary source for the physical, psychological and treatment-related support for people with mental illness in many low-income countries. ${ }^{21}$ The positive effect of family interventions on clinical and functional outcomes of people with bipolar disorder ${ }^{13,23}$ may be even greater in this setting. However, previous work from Ethiopia has also highlighted the differing priorities of people with mental health conditions and their caregivers, underlining the need to ensure that the person with bipolar disorder remains at the centre of care.

People with bipolar disorder and their caregivers, community leaders and health professionals had different perspectives for improving long-term outcomes. People with bipolar disorder and their caregivers prioritised social and functional outcomes, such as educational impact, stigma and productivity, ${ }^{22}$ as the most important long-term outcome. However, community leaders acknowledged the difficulties of social engagement related to public stigma and emphasised stigma reduction as a long termoutcome, ${ }^{43}$ as they viewed these as important barriers to reintegration into the community. Health professionals identified the improved quality of life of people with bipolar disorder and reduced caregiver burden as long-term outcomes. This may also reflect the view of health professionals with limited expertise regarding the longer-term outcome of bipolar disorder. These different perspectives are relevant and point to the need for a multi-layered intervention package beyond a health facility-based intervention to address multidimensional needs. Further development work to address these multisectoral and multidimensional issues is essential.

In LMICs like Ethiopia, the treatment gap for mental illness is around $90 \%$. $^{3}$ The World Health Organization has recommended the integration of mental health services within PHC settings to address unmet mental health needs. ${ }^{27}$ In our study, participants supported the delivery of the intervention by $\mathrm{PHC}$ workers to facilitate its future integration within routine care. Although the participants were concerned about the possible work burden on PHC workers, they considered that training would enhance PHC providers' skills, interest and confidence in providing the intervention, which could in turn reduce the feeling of work burden. Additionally, group intervention was noted to be less feasible for this rural setting, where there is inadequate local transportation and it is difficult to identify a convenient time for group intervention. ${ }^{44}$

\section{Implications of the study}

This intervention was developed based on previous evidence and by involving mental health specialists with clinical and research experience, PHC workers, people with bipolar disorder and their caregivers, and community leaders. The approach focused on intervention content, manual and leaflet preparation, language and delivery strategies. This improved stakeholders buy-in, ${ }^{34}$ ensuring that this intervention will be feasible and acceptable. A pilot test to assess the feasibility, fidelity and acceptability in routine clinical settings, and to ensure the scalability and preliminary efficacy of the developed psychological intervention, is warranted.

\section{Strengths and limitations}

The main strength of this study is that we began the intervention development by synthesising evidence on the efficacy of the psychological intervention in LMICs and conducting formative work. Our study involved diverse stakeholder groups, which can ensure the intervention's local appropriateness through stakeholder buy-in and direct inputs. Although we sought to reduce the impact of power imbalances in the ToC workshops by conducting separate workshops with groups of similar stakeholders, a residual power imbalance (e.g. between caregivers and people with bipolar disorder) may still have affected the content of what participants felt comfortable to expressing. The skilled facilitator sought to address this by actively seeking out the views of people with bipolar disorder. However, even skilled facilitation did not fully address the power imbalance; therefore, future studies may consider capacity-building training to enhance participants' capacity before conducting ToC interventions. Finally, we brought all stakeholder groups together at the end of the study, which allowed us to ensure that the views of all participants were incorporated. However, some participants in the group of people with bipolar disorder, their caregivers and the community, struggled to understand the visual presentation of the ToC map, especially the final ToC map that included the merged ToC map. This could be because of the low literacy rate in a rural community, the lack of experience with intervention development and the technical elements of the ToC map. Regarding reviewing the manual, people with bipolar disorder and their caregivers were not involved in reviewing the manual, unlike PHC workers. However, in the final ToC workshop, the intervention content and the number and frequency of the sessions were discussed and approved by all ToC participants, including people with bipolar disorder and their caregivers. Additionally, we plan to evaluate the feasibility and acceptability of the actual implementation of the intervention and address any gaps that are identified in the next step.

Although financial problems were repeatedly reported in our qualitative study ${ }^{22}$ and ToC workshops, economic problems were not directly targeted with our intervention. However, we do anticipate indirect benefits to financial status through the problemsolving techniques and change to the anticipated outcomes, like reduced symptom severity and caregiver burden, improved wellbeing of people with bipolar disorder and reduced stigma ${ }^{45}$ However, further studies are needed to determine better ways to reduce financial problems.

In conclusion, the mixed approach that we have used to develop the intervention, including a systematic review, qualitative study, expert workshop and ToC workshops, enabled us to understand the needs and priorities of people with bipolar disorder and their caregivers, and identify intervention components. The ToC workshop helped us to develop the ToC map, which includes an intervention component, underlying assumptions and preconditions for its effective implementation. Finally, the methods enabled us to develop a culturally appropriate, individual psychosocial intervention that can be delivered in five sessions in the PHC setting. This intervention needs to be tested for feasibility, acceptability and effectiveness before wider implementation.

Mekdes Demissie (D), WHO Collaborating Centre for Mental Health Research and Capacity Building, Department of Psychiatry, School of Medicine, College of Health Sciences, Addis Ababa University, Ethiopia; and School of Nursing and Midwifery, College of Health Sciences and Medicine, Haramaya University, Ethiopia; Charlotte Hanlon (D), WHO Collaborating Centre for Mental Health Research and Capacity Building,

Department of Psychiatry, School of Medicine, College of Health Sciences, Addis Ababa University, Ethiopia; Centre for Innovative Drug Development and Therapeutic Studies for Africa (CDT-Africa), College of Health Sciences, Addis Ababa University, Ethiopia; and Centre for Global Mental Health, Department of Health Services and Population Research, Institute of Psychiatry, Psychology and Neuroscience, King's College London, UK; Lauren Ng, Department of Psychology, University of California, Los Angeles, USA; Rosie Mayston (D), King's Global Health Institute, Department of Global Health and 
Social Medicine, King's College London, UK; Sisay Abayneh, WHO Collaborating Centr for Mental Health Research and Capacity Building, Department of Psychiatry, School of Medicine, College of Health Sciences, Addis Ababa University, Ethiopia;

Abebaw Fekadu, WHO Collaborating Centre for Mental Health Research and Capacity Building, Department of Psychiatry, School of Medicine, College of Health Sciences, Addis Ababa University, Ethiopia: Centre for Innovative Drug Development and Therapeutic Ababa Un Sthiopia; Department of Global Health and Infection, Brighton and Sussex Medical School, University of Sussex, UK; and Centre for Affective Disorders, Department of Psychologica Medicine, Institute of Psychiatry, Psychology and Neuroscience, King's College London, UK

Correspondence: Mekdes Demissie. Email: mekdesdemissie2016@gmail.com

First received 1 Mar 2021, final revision 10 Aug 2021, accepted 12 Aug 2021

\section{Supplementary material}

Supplementary material is available online at https://doi.org/10.1192/bjo.2021.999

\section{Data availability}

The authors confirm that the data supporting the findings of this study are available within the article and its supplementary materials.

\section{Author contributions}

M.D., A.F. and C.H. designed the study. M.D. led the study, and drafted and revised the manuscript with supervision from A.F., C.H., R.M. and L.N. The mental health experts workshop was led by M.D. and A.F. The ToC workshops were run by M.D. and S.A. M.D. and C.H. conducted the analysis. A.F., C.H., R.M., S.A. and L.N. edited the paper. All authors read and approved the final manuscript.

\section{Funding}

This work was supported through the DELTAS Africa Initiative (grant DEL-15-01). The DELTAS Africa Initiative is an independent funding scheme of the African Academy of Sciences' Alliance for Accelerating Excellence in Science in Africa (AESA), and is supported by the New Partnership for Africa's Development Planning and Coordinating Agency (NEPAD Agency) with funding from the Wellcome Trust (grant DEL-15-01) and the UK Government The views expressed in this publication (manual) are those of the authors and not necessarily those of AAS, NEPAD Agency, Wellcome Trust or the UK Government. Addis Ababa University also supported this study financially. None of the funding bodies had a role in study design, data collection and analysis, decision to publish or preparation of the manuscript. The authors acknowledge the contributions of all stakeholders participated in the qualitative study, mental health expert workshop and the ToC workshops. We would like to thank Sodo District Health Office and PRIME project field coordinators who invested their time and effort in facilitating the workshops.

\section{Declaration of interest}

Professor Abebaw Fekadu is a memebr of the Editorial Board but he was not involved in the peer review or decision making process of the paper.

\section{References}

1 Merikangas KR, Jin R, He J-P, Kessler RC, Lee S, Sampson NA, et al. Prevalence and correlates of bipolar spectrum disorder in the world mental health survey initiative. Arch Gen Psychiatry 2011; 68(3): 241-51.

$2 \mathrm{He} \mathrm{H}$, Hu C, Ren Z, Bai L, Gao F, Lyu J. Trends in the incidence and DALYS of bipolar disorder at global, regional, and national levels: results from the Global Burden of Disease Study 2017. J Psychiatr Res 2020; 125: 96-105.

3 Fekadu A, Hanlon C, Thornicroft G, Lund C, Kaaya S, Alem A, et al. Care for bipolar disorder in LMICs needs evidence from local settings. Lancet Psychiatry 2015; 2(9): 772-3.

4 Fekadu A, Medhin G, Kebede D, Alem A, Cleare AJ, Prince M, et al. Excess mortality in severe mental illness: 10-year population-based cohort study in rural Ethiopia. Br J Psychiatry 2015; 206(4): 289-96.

5 Kebede D, Alem A, Shibire T, Deyassa N, Negash A, Beyero T, et al. Symptomatic and functional outcome of bipolar disorder in Butajira, Ethiopia. J Affect Disord 2006; 90(2): 239-49.

6 Fekadu A, Shibre T, Alem A, Kebede D, Kebreab S, Negash A, et al. Bipolar disorder among an isolated island community in Ethiopia. J Affect Disord 2004; 80(1): 1-10

7 Fekadu A, Kebede D, Alem A, Fekadu D, Mogga S, Negash A, et al. Clinical outcome in bipolar disorder in a community-based follow-up study in Butajira, Ethiopia. Acta Psychiatr Scand 2006; 114(6): 426-34.

8 Belete $\mathrm{H}$. Leveling and abuse among patients with bipolar disorder at psychiatric outpatient departments in Ethiopia. Ann Gen Psychiatry 2017; 16: 29.
9 Addisu F, Wondafrash M, Chemali Z, Dejene T, Tesfaye M. Length of stay of psychiatric admissions in a general hospital in Ethiopia: a retrospective study. Int J Ment Health Syst 2015; 9: 13

10 Ayano G, Duko B. Relapse and hospitalization in patients with schizophrenia and bipolar disorder at the St Amanuel Mental Specialized Hospital, Addis Ababa, Ethiopia: a comparative quantitative cross-sectional study. Neuropsychiatr Dis Treat 2017: 13: 1527-31.

11 Saxena S, Thornicroft G, Knapp M, Whiteford $\mathrm{H}$. Resources for mental health scarcity, inequity, and inefficiency. Lancet 2007; 370(9590): 878-89.

12 Colom F, Vieta E, Tacchi M, Sánchez-Moreno J, Scott J. Identifying and improving non-adherence in bipolar disorders. Bipolar Disord 2005; 7: 24-31.

13 Fristad MA, MacPherson HA. Evidence-based psychosocial treatments for child and adolescent bipolar spectrum disorders. J Clin Child Adolesc Psychol 2014; 43(3): 339-55.

14 Sylvia LG, Chang WC, Kamali M, Tohen M, Kinrys G, Deckersbach T, et al. Sleep disturbance may impact treatment outcome in bipolar disorder: a preliminary investigation in the context of a large comparative effectiveness trial. J Affect Disord 2018; 225: 563-8.

15 Levy B، Tsoy E، Brodt M, Petrosyan K, Malloy M. Stigma, social anxiety, and illness severity in bipolar disorder. Implications Treat 2015; 27(1): 55-64.

16 Koenders M, Giltay E, Spijker A, Hoencamp E, Spinhoven P, Elzinga B. Stressful life events in bipolar I and II disorder: cause or consequence of mood symptoms? J Affect Disord 2014; 161: 55-64

17 Messer T, Lammers G, Müller-Siecheneder F, Schmidt R-F, Latifi S. Substance abuse in patients with bipolar disorder: a systematic review and meta-analysis. Psychiatry Res 2017; 253: 338-50.

18 Hanlon C. Next steps for meeting the needs of people with severe mental illness in low-and middle-income countries. Epidemiol Psychiatr Sci 2017; 26 (4): $348-54$

19 Gaudiano BA, Weinstock LM, Miller IW. Improving treatment adherence in patients with bipolar disorder and substance abuse: rationale and initial development of a novel psychosocial approach. J Psychiatr Pract 2011; 17(1): 5-20.

20 World Health Organization. mhGAP Intervention Guide for Mental, Neurological and Substance Use Disorders in Non-Specialized Health Settings: Mental Health Gap Action Programme (mhGAP). World Health Organization, 2016 (https:// apps.who.int/iris/bitstream/handle/10665/250239/9789241549790-eng.pdf).

21 Mall S, Hailemariam M, Selamu M, Fekadu A, Lund C, Patel V, et al. 'Restoring the person's life': a qualitative study to inform development of care for people with severe mental disorders in rural Ethiopia. Epidemiol Psychiatr Sci 2017; 26 (1): 43-52.

22 Demissie M, Hanlon C, Birhane R, Ng L, Medhin G, Fekadu A. Why doesn't God say "enough"? Experience of living with bipolar disorder in rural Ethiopia. Soc Sci Med 2021; 270: 113625

23 Miziou S, Tsitsipa E, Moysidou S, Karavelas V, Dimelis D, Polyzoidou V, et al. Psychosocial treatment and interventions for bipolar disorder: a systematic review. Ann Gen Psychiatry 2015; 14: 19.

24 Patel V, Chowdhary N, Rahman A, Verdeli H. Improving access to psychological treatments: lessons from developing countries. Behav Res Ther 2011; 49(9): 523-8.

25 Bruckner TA, Scheffler RM, Shen G, Yoon J, Chisholm D, Morris J, et al. The mental health workforce gap in low-and middle-income countries: a needs-based approach. Bull World Health Organ 2011; 89: 184-94.

26 Van Ginneken N, Tharyan P, Lewin S, Rao GN, Meera S, Pian J, et al. Non-specialist health worker interventions for the care of mental, neurological and substance-abuse disorders in low-and middle-income countries. Cochrane Database Syst Rev 2013; 11: CD009149.

27 World Health Organization. Integrating Mental Health Into Primary Care: A Global Perspective. World Health Organization, 2008 (https://www.who.int/ mental health/resources/mentalhealth PHC 2008.pdf).

28 Fekadu A, Hanlon C, Medhin G, Alem A, Selamu M, Giorgis TW, et al Development of a scalable mental healthcare plan for a rural district in Ethiopia. Br J Psychiatry 2016; 208(suppl 56): s4-12.

29 Demissie M, Hanlon C, Birhane R, Ng L, Medhin G, Fekadu A. Psychological interventions for bipolar disorder in low-and middle-income countries: systematic review. BJPsych Open 2018; 4(5): 375-84.

30 Griner D, Smith TB. Culturally adapted mental health intervention: a meta-analytic review. Psychotherapy 2006; 43(4): 531-48.

31 De Silva MJ, Breuer E, Lee L, Asher L, Chowdhary N, Lund C, et al. Theory of change: a theory-driven approach to enhance the Medical Research Council's framework for complex interventions. Trials 2014; 15: 267.

32 Central Statistical Agency of Ethiopia. Population Projection of Ethiopia for All Regions at Wereda Level from 2014-2017. Central Statistical Agency of Ethiopia, 2013 (https://www.statsethiopia.gov.et/population-projection/).

33 Hanlon C, Alem A, Medhin G, Shibre T, Ejigu DA, Negussie H, et al. Task sharing for the care of severe mental disorders in a low-income country (TaSCS): 
study protocol for a randomised, controlled, non-inferiority trial. Trials 2016; 17: 76

34 Breuer E, De Silva MJ, Fekadu A, Luitel NP, Murhar V, Nakku J, et al. Using workshops to develop theories of change in five low and middle income countries: lessons from the programme for improving mental health care (PRIME). Int J Ment Health Syst 2014; 8: 15.

35 Duncan E, O'cathain A, Rousseau N, Croot L, Sworn K, Turner KM, et al. Guidance for reporting intervention development studies in health research (GUIDED): an evidence-based consensus study. BMJ Open 2020; 10 (4): e033516

36 Ravitz P, Wondimagegn D, Pain C, Araya M, Alem A, Baheretibeb Y, et al. Psychotherapy knowledge translation and interpersonal psychotherapy: using best-education practices to transform mental health care in Canada and Ethiopia. Am J Psychother 2014; 68(4): 463-88.

37 Patel V, Hanlon C. Where There Is No Psychiatrist: A Mental Health Care Manual. Cambridge University Press, 2018.

38 Miklowitz DJ, O'Brien MP, Schlosser DA, Zinberg JL, De Silva S, George EL, et al. Clinicians' Treatment Manual for Family-Focused Therapy for Early-Onset Youth and Young Adults (FFT-EOY). August 28, 2012 (https://www.nasmhpd. org/sites/default/files/FFT-EOY_Manual_82812.pdf).

39 Colom F, Vieta E. Psychoeducation Manual for Bipolar Disorder. Cambridge University Press, 2006.
40 Lam DH, Jones SH, Hayward P. Cognitive Therapy for Bipolar Disorder: A Therapist's Guide to Concepts, Methods and Practice. John Wiley \& Sons, 2010.

41 Cuijpers $\mathrm{P}$, Reijnders $\mathrm{M}$, Huibers MJ. The role of common factors in psychotherapy outcomes. Annu Rev Clin Psychol 2019; 15: 207-31.

42 O'Brien N, Heaven B. Integrating evidence from systematic reviews, qualitative research, and expert knowledge using co-design techniques to develop a webbased intervention for people in the retirement transition. J Med Internet Res 2016; 18(8): e210.

43 Semrau M, Evans-Lacko S, Koschorke M, Ashenafi L, Thornicroft G. Stigma and discrimination related to mental illness in low-and middle-income countries. Epidemiol Psychiatr Sci 2015; 24(5): 382-94.

44 Chatterjee S, Chowdhary N, Pednekar S, Cohen A, Andrew G, Andrew G, et al. Integrating evidence-based treatments for common mental disorders in routine primary care: feasibility and acceptability of the MANAS intervention in Goa, India. World Psychiatry 2008; 7(1): 39-46.

45 Shibre T, Medhin G, Teferra S, Wakwoya A, Berhanu E, Abdulahi A, et al. Predictors of carer-burden in schizophrenia: a five-year follow-up study in Butajira, Ethiopia. Ethiop Med J 2012; 50(2): 125-33.

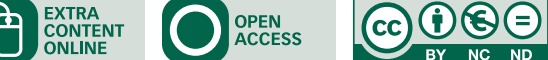

